\title{
Optimal Estimation of Parameters for an HIV Model
}

\author{
Danna Sun, Zhaoying Jiang, Ziku Wu* \\ College of Science and Information, Qingdao Agricultural University, Qingdao, China \\ Email: msj1958@126.com, zkwu1968@126.com
}

Received 2013

\begin{abstract}
An HIV model was considered. The parameters of the model are estimated by adjoint dada assimilation method. The results showed the method is valid. This method has potential application to a wide variety of models in biomathematics.
\end{abstract}

Keywords: HIV Model; Optimal Estimation; Adjoint Data Assimilation

\section{Introduction}

One of the worst diseases in the world is AIDS (Acquired Immunity Deficiency Syndrome). It is caused by the human immunodeficiency virus (HIV). There has been much interest recently in mathematical models of HIV. During the last decade, many scholars have done a great of work about HIV model ([1-6]). We consider a model which presented by Henry et al. ([1]), which describes the interaction of HIV and the immune system of the body. In this model, the variables are uninfected CD4 + T-cells, infected such cells and free virus, whose densities at time $t$ are denoted respectively by $x(t), y(t)$ and $v(t)$. These quantity satisfy

$$
\left\{\begin{array}{l}
\frac{d x}{d t}-s+\mu x+\beta x y=0 \\
\frac{d y}{d t}-\beta x y+\alpha y=0 \\
\frac{d v}{d t}-c y+\gamma v=0
\end{array}\right.
$$

with initial values $x(0)>0, y(0) \geq 0$ and $v(0)>0$. Here $s, \mu, \beta, \alpha, c$ and $\gamma$ are model parameters which interpreted as follows:

$s:$ is the rate of production of CD4 + T-cells

$\mu$ : is their per capita death rate

$\beta$ : is the rate of infection of CD4 + T-cells by virus

$\alpha$ : is the per capita rate of disappearance of infected cells

$c$ : is the rate of production of virus by infected cells

$\gamma:$ is the death rate of virus particles.

It's impossible to get the above model's analytic solutions due to its strong nonlinearity. Thus we computed its solutions using Runge-Kutta methods of order 4. This

${ }^{*}$ Corresponding author. was done with initial Thus we computed its solutions using Runge-Kutta methods of order 4 . This was done with initial $x(0)=200, y(0)=0$ and $v(0)=1$, and typical parameters values $s=0.272, \mu=0.00136, \beta=$ $0.00027, \alpha=0.33, c=50$ and $\gamma=2$. The time series of uninfected CD4+T-cells and infected CD4 + T-cells are listed in Figures 1 and 2, respectively. The peak values exist round 20 days. The phase portraits are shown in Figure 3 for a time period of 200 days and it can be seen that the orbits are quite close.

These model parameters are chosen to be consistent with those in the model, whose values may not be very well known. There has recently been considerable interest in the inverse problem of determining such values by incorporating measured data into the numerical model. The adjoint assimilation method involves minimizing a certain cost function which consists basically of a norm of the difference between the computed and the observed values of the measured variables. The purpose of the

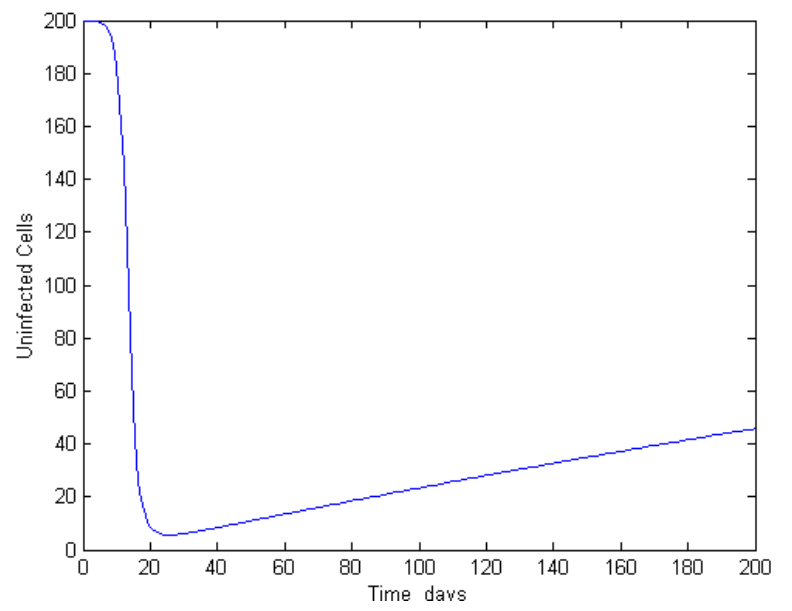

Figure 1. The time series of uninfected CD4 + T-cell. 


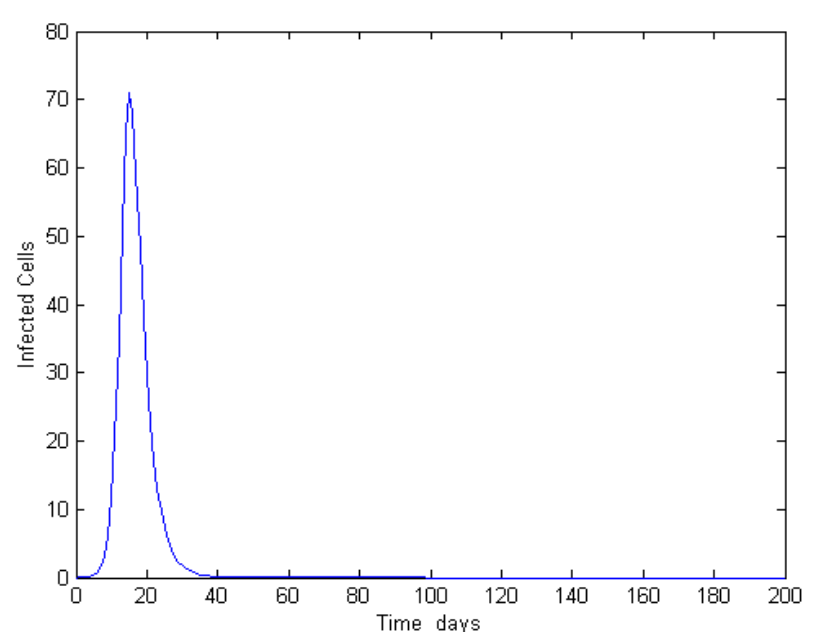

Figure 2. The time series of infected CD4 + T-cell.

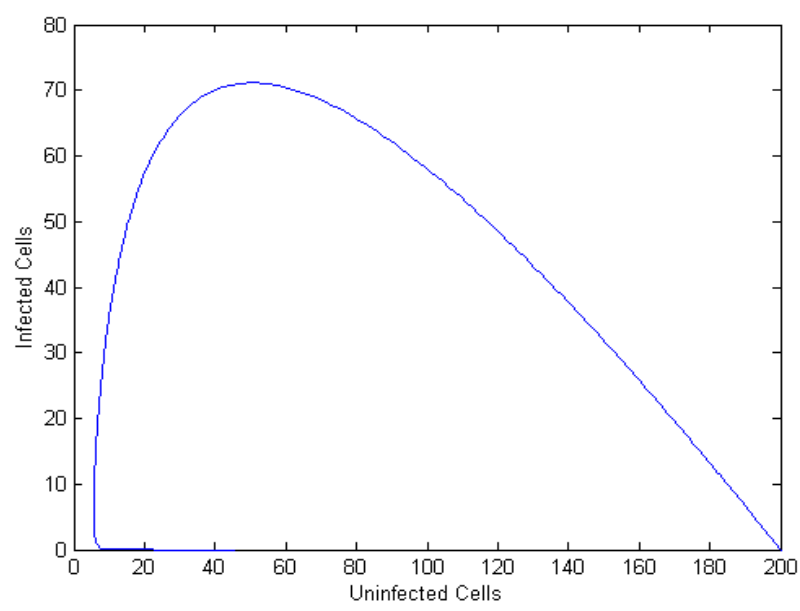

Figure 3. The phase portraits.

present paper is to estimate the model parameters $(\mu, \alpha, \lambda)$ by constructing the adjoint model. The basis of the method is to minimize the cost function which is equal to a norm of difference between the computed and the observed data. An algorithm is obtained, via so-called adjoint equation, for construction of the gradient of the function with respect to the parameters.

In Section 2 we describe the adjoint assimilation method. A detailed discussion of the numerical tests results is given in Section 3. Section 4 summarizes the results and conclusions.

\section{The Adjoint Numerical Model}

For parameter estimation and data assimilation, differences between predicted and measured values of these variables must be quantified by a single misfit number, the objective function. A lot of options are available for choosing the misfit function. In this study, we choose the classical least-squares approach. However, the results from above governing equations are not good as with the observed values. The error between observed and model calculated can be defined as

$$
J=\frac{1}{2} \int_{0}^{T}\left(w_{1}\left(x-x^{o}\right)^{2}+w_{2}\left(y-y^{o}\right)^{2}+w_{3}\left(v-v^{o}\right)^{2}\right) d t
$$

Where $x^{o}, y^{o}$ and $v^{o}$ present observation of $x, y$ and $v$, respectively. $w_{1}, w_{2}$ and $w_{3}$ are weight coefficients and $[0, T]$ stands for assimilation window.

In order to get the adjoint Equations of (1), we introduce the adjoint control variables and Lagrange function:

$$
L=J+\int_{0}^{T}(X L x+Y L y+V L v) d t
$$

where $X, Y$ and $V$ are adjoint variables. $L x, L y$, and $L v$ are the left hand part of Equations (1). It is easy to get the following adjoint equations:

$$
\left\{\begin{array}{l}
-\frac{d X}{d t}+\mu X+\beta v(X-Y)+w_{1}\left(x-x^{o}\right)=0 \\
-\frac{d Y}{d t}+\alpha Y-c V+w_{2}\left(y-y^{o}\right)=0 \\
-\frac{d V}{d t}+\gamma V+\beta x(X-Y)+w_{3}\left(v-v^{o}\right)=0
\end{array}\right.
$$

Now the parameters $\mu, \alpha$ and $\gamma$ are unknown, which need to estimate by the adjoint assimilation method. The numerical scheme of the adjoint Equation (4) are the same as Equation (1), however it needs to integrate backward, and its initial conditions are set to zeros, that is

$$
X(t)=0, Y(T)=0, V(T)=0
$$

The gradients can be easily obtained through Equation (3), which are

$$
\left\{\begin{array}{l}
\frac{\partial J}{\partial \mu}=\int_{0}^{T} x X d t \\
\frac{\partial J}{\partial \alpha}=\int_{0}^{T} y Y d t \\
\frac{\partial J}{\partial \gamma}=\int_{0}^{T} v V d t
\end{array}\right.
$$

For the sake of convenience, we denotes

$$
\begin{gathered}
P=(\mu, \alpha, \gamma)^{\prime} \\
\nabla_{J} P=\left(\frac{\partial J}{\partial \mu}, \frac{\partial J}{\partial \alpha}, \frac{\partial J}{\partial \gamma}\right)^{\prime}
\end{gathered}
$$

Having determined the gradients which respect to the unknown parameters, we can perform the minimization by the descend method, the modified parameters are

$$
p a \Leftarrow p a-\lambda \times g d
$$

where $\lambda$ is the optimal step. 


\section{Numerical Tests}

In real applications, the model must be calibrated against experimental data. In this numerical study, however, a twin experiment is carried out: a reference solution is generated with the model itself using the parameters the same as in Section I.

In this part, we design 3 numerical tests, 2 tests with random error in the synthetic data, which denoted TX1 with no errors, TX2 with 1\% errors, TX3 with $3 \%$ errors, respectively. Only the infected CD4 + T-cells observed data are valid, that is $w_{1}=w_{3}=0$. The first guess of them are $1.0 \mathrm{E}-3,0.2$ and 1.5 , respectively. The observed dada are plotted in Figure 4. The numerical results listed in Table 1. The cost function descent curve showed in Figure 5.

\section{Summary and Discussion}

Adjoint data assimilation method is employed to estimate the parameters of a kind of HIV model. The parameters estimated are $\mu, \alpha$ and $\gamma$. The method is based on an optimal control approach where by a cost function measuring the discrepancies between numerical computed and measured is minimized, subject to constrains consisting of equations of the model. The numerical scheme used is the forth order Runge-Kutta method. In order to test the validity of this method, we have designed several experiments. The results showed the method is valid. The research results are useful to understand the HIV model and helpful to establish a robust and effective HIV control and prediction.

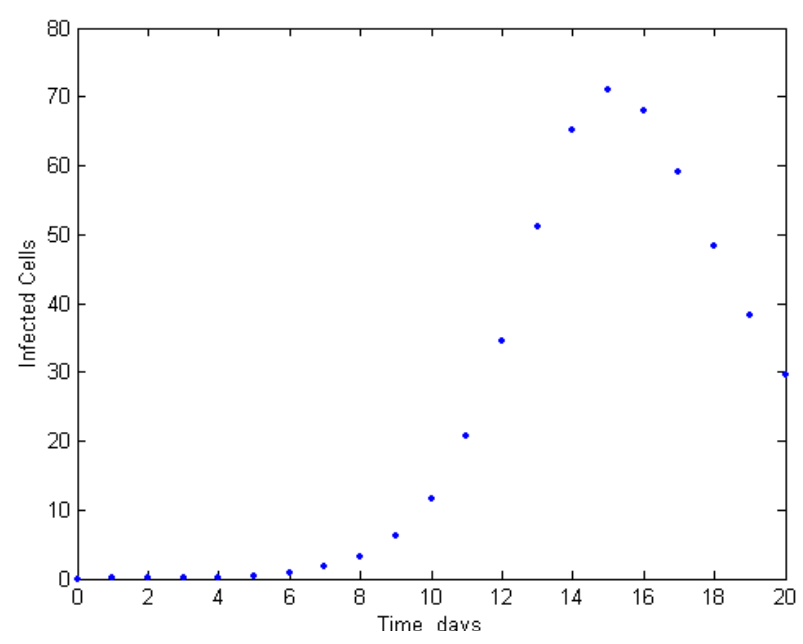

Figure 4. The observed data.

Table 1. Estimated parameters values.

\begin{tabular}{cccc}
\hline & TX1 & TX2 & TX3 \\
\hline $\boldsymbol{\mu}$ & $1.371 \mathrm{E}-3$ & $1.412 \mathrm{E}-3$ & $1.501 \mathrm{E}-3$ \\
$\alpha$ & 0.328 & 0.343 & 0.352 \\
$\gamma$ & 1.996 & 2.051 & 2.132 \\
\hline
\end{tabular}

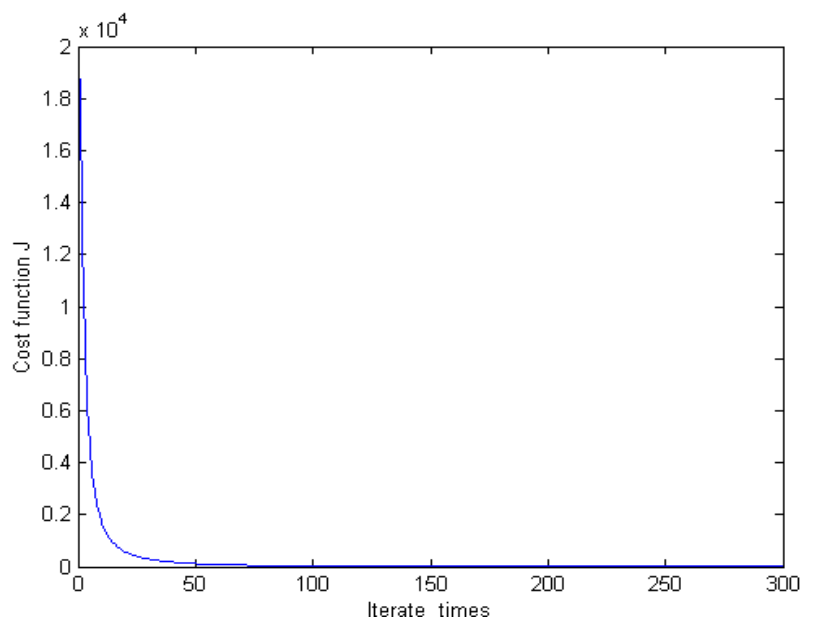

Figure 5. The cost function descent curve.

\section{Acknowledgements}

This work is supported by "A Project of Shandong Province Higher Educational Science and Technology Program (J09LA12)" and "Shandong Provincial Natural Science Foundation (ZR2009AL012)”.

\section{REFERENCES}

[1] H. C. Tuckwell and F. Y. M. Wan, "On the Behavior of Solutions in Viral Dynamical Models,” BioSystems, Vol. 73, 2004, pp. 157-161. http://dx.doi.org/10.1016/j.biosystems.2003.11.004

[2] J. F. Zhang and X. H. Xia, "Identifiability Problems of Time-Delay HIV Models," Proceedings of the 17th World Congress, The International Federation of Automatic Control, Seoul, 2008, pp. 283-288.

[3] R. V. Culshaw, S. G. Ruan and R. J. Spiteri, “Optimal HIV Treatment by Maximising Immune Response,” Mathematical Biology, Vol. 48, 2004, pp. 545-562. http://dx.doi.org/10.1007/s00285-003-0245-3

[4] C. Myburgh and K. H. Wong, "Computational Control of an HIV Model,” Annals of Operations Research, Vol. 133, 2005, pp. 277-283. http://dx.doi.org/10.1007/s10479-004-5038-6

[5] J. M. Hyman and J. Li, "The Reproductive Number for an HIV Model with Differential Infectivity and Staged Progression,” Linear Algebra and Its Applications, Vol. 398, 2005, pp. 101-116.

http://dx.doi.org/10.1016/j.laa.2004.07.017

[6] H.-D. Kwon, "Optimal Treatment Strategies Derived from a HIV Model with Drug-Resistant Mutants,” Applied Mathematics and Computation, Vol. 188, 2007, pp. 11931204. http://dx.doi.org/10.1016/j.amc.2006.10.071 